\title{
Upper limits on the WIMP annihilation cross section from a joint analysis of dwarf spheroidal satellite galaxy observations with the MAGIC telescopes
}

\author{
Camilla Maggio, ${ }^{a *}$ Daniel Kerzsberg, ${ }^{b}$ Daniele Ninci ${ }^{c}$ and Vincenzo Vitale ${ }^{d}$ on behalf \\ of the MAGIC Collaboration \\ (a complete list of authors can be found at the end of the proceedings) \\ ${ }^{a}$ Departament de Física \& CERES-IEEC, Universitat Autónoma de Barcelona, \\ Bellaterra, Spain \\ ${ }^{b}$ Institut de Fisica d'Altes Energies \& The Barcelona Institute of Science and Technology, \\ Bellaterra, Spain \\ ${ }^{c}$ Institut de Fisica d'Altes Energies \& The Barcelona Institute of Science and Technology, \\ Bellaterra, Spain \\ ${ }^{d}$ INFN Roma Tor Vergata, \\ Roma, Italy \\ E-mail: cmaggio.astro@outlook.com, dkerzsberg@ifae.es, dninci@ifae.es, \\ vvitale@roma2.infn.it
}

Dwarf spheroidal galaxies (dSphs) are among the best candidates to perform indirect searches for DM, having the highest known mass-to-light ratio and being free of astrophysical gamma-ray emitting sources. The Major Atmospheric Gamma Imaging Cherenkov (MAGIC) telescopes, located on the Canary Island of La Palma, have observed a fair amount of optimal dSphs in the recent years. This is the outcome of diversifying the observation strategy in order to avoid possible biases in target selection and to improve previous results. In this contribution we will report on new MAGIC results obtained from 52.1 hours of observation of the Draco dSph in 2018 and 49.5 hours of the Coma Berenices dSph in 2019. We will also present the results of a joint analysis of Draco and Coma Berenices dSphs with other dSphs observed by MAGIC so far. The selected dataset accounts for 354.4 hours of good quality data, resulting in one of the largest dSphs samples ever collected by an array of Cherenkov telescopes. This allows us to derive the most constraining limits from dSphs, among Cherenkov telescopes, on the WIMP annihilation cross section for different annihilation channels in the WIMP mass range $70 \mathrm{GeV}$ to $100 \mathrm{TeV}$.

$37^{\text {th }}$ International Cosmic Ray Conference (ICRC 2021)

July 12th - 23rd, 2021

Online - Berlin, Germany

\footnotetext{
${ }^{*}$ Presenter
} 


\section{Introduction}

Dark Matter (DM) is assumed to be present in all the Universe and to be gravitationally bound in overdensity structures, the so-called DM halos. Following the hierarchical bottom-up structure formation scenario [1], these halos usually host astrophysical objects, such as galaxies or clusters of galaxies. The dwarf spheroidal galaxies (dSphs) satellite of the Milky Way are one of the best targets to perform indirect DM searches. These objects are almost free of conventional astrophysical background and are relatively close to us, orbiting our Galaxy.

Indirect DM searches with Imaging Atmospheric Cherenkov Telescopes (IACTs), such as the Florian Göebel Major Atmospheric Gamma Imaging Cherenkov (MAGIC) telescopes [2], select the objects to study on the basis of their DM content and vicinity to the Earth. These criteria make dSphs one of the targets most investigated, in particular in the case of searches for DM annihilation.

The Weakly Interacting Massive Particle (WIMP) [3] is the DM particle candidate preferred for IACTs searches, having a mass in the range $10 \mathrm{GeV}$ to $100 \mathrm{TeV}$ [4] and emitting gamma rays among the products of annihilation/decay. Thus, models of WIMP DM annihilation/decay and the DM distribution are the main ingredients taken into account when performing DM searches with IACTs. The MAGIC telescopes are a system of two IACTs operating simultaneously, in the so-called stereo mode, placed in the Observatorio del Roque de los Muchachos, on the Canary Island of La Palma (Spain). Since their first light, they observed dSphs in search for a gamma-ray signal of WIMP DM annihilation, but no hint of DM has been found yet. In this proceeding, we present the data samples of the new targets observed by MAGIC in the multi-year dSphs diversification program and the results of the analysis of the single $\mathrm{dSphs}$, followed by the results of the analysis of a combined data sample, in which dSphs previously observed by MAGIC are also included.

\section{Dwarf spheroidal galaxies data samples}

The new MAGIC multi-year dSphs observation diversification program aims to detect hints of DM signals from promising dSphs targets in the Northern Hemisphere, or, in case of no detection, to improve the current limits on the velocity-averaged cross-section of WIMPs. This is done by differentiating the targets and accumulating data, in order to have a larger data sample that permits to reduce the systematic uncertainties and improve the results.

For this purpose, the two dSphs Draco and Coma Berenices were observed in 2018 and 2019 for a total effective time of $52.1 \mathrm{~h}$ and $49.5 \mathrm{~h}$ respectively. The dSphs were selected after a ranking of the dSphs presented in [5], taking into account the DM content, its uncertainty, and their distance from us, excluding the dSphs previously observed by MAGIC.

The DM analysis has been performed independently for each data sample acquired, as described in Section 3. In addition, previously observed Segue 1 and Ursa Major II dSphs data samples have been combined to the new data, resulting in a total amount of $354.4 \mathrm{~h}$ of good quality data. The results of the analysis on this overall data sample, together with the other results, are presented in Section 4. In Table 1, the effective observation time of the dSphs included in the combined data sample is presented. The order in which the dSphs are listed follows the year of observation, from the oldest observation to the newest one. 
Table 1: dSphs effective observation times (after data quality selection).

\begin{tabular}{cc} 
Target & $T_{\text {obs }}[\mathrm{h}]$ \\
\hline Segue 1 & 157.9 \\
Ursa Major II & 94.8 \\
Draco & 52.1 \\
Coma Berenices & 49.5 \\
\hline
\end{tabular}

\section{DM likelihood analysis}

When searching for a DM annihilation signal with IACTs, the measured quantity is the flux of gamma rays emitted by the target of interest. This flux can be written as the product of two terms, a particle physics $(P P-)$ factor, containing the particle physics properties of the considered DM particle candidate, and an astrophysical (or $J$-) factor, containing information of the DM spatial distribution of the target:

$$
\frac{d \Phi(\Delta \Omega)}{d E}=\frac{1}{4 \pi} \frac{\left\langle\sigma_{\mathrm{ann}} v\right\rangle}{2 m_{\mathrm{DM}}^{2}} \frac{d N}{d E} \times \int_{\Delta \Omega} d \Omega^{\prime} \int_{\text {l.o.s. }} d l \rho^{2}\left(l, \Omega^{\prime}\right) .
$$

On the right-hand-side of the equation, one can find the geometrical factor $\frac{1}{4 \pi}$, the $P P$-factor, described by the velocity-averaged cross-section $\left\langle\sigma_{\mathrm{ann}} v\right\rangle$, the DM mass $m_{\mathrm{DM}}$ and the gamma-ray spectrum $\frac{d N}{d E}=\sum_{i=1}^{n} \mathrm{Br}_{i} \frac{d N_{i}}{d E}$ of $n$ possible annihilation channels weighted by the corresponding branching ratios $\mathrm{Br}_{i}$, and the $J$-factor, written as the integral over the solid angle $\Delta \Omega$ and the line-of-sight of the squared DM density $\rho$ of the target.

In order to compute the velocity-averaged cross-section of a WIMP particle, after the standard data reduction achieved by the MAGIC analysis software MARS [6], a binned likelihood analysis is performed on the data. The likelihood function, per target $t$ and pointing direction $i$, as a function of the dataset $\mathcal{D}$, is expressed as:

$$
\begin{aligned}
& \mathcal{L}_{t i}\left(\left\langle\sigma_{\mathrm{ann}} v\right\rangle ; v \mid \mathcal{D}\right)=\mathcal{L}\left(\left\langle\sigma_{\mathrm{ann}} v\right\rangle ; J_{t},\left\{b_{t i j}\right\}_{j=1, \ldots, N_{\mathrm{bins}},}, \tau_{t i} \mid\left(N_{\mathrm{ON}, t i j}, N_{\mathrm{OFF}, t i j}\right)_{j=1, \ldots, N_{\mathrm{bins}}}\right) \\
& =\prod_{j=1}^{N_{\mathrm{bins}}}\left[\frac{\left(g_{t i j}\left(\left\langle\sigma_{\mathrm{ann}} v\right\rangle, J_{t}\right)+b_{t i j}\right)^{N_{\mathrm{ON}, t i j}}}{N_{\mathrm{ON}, t i j} !} e^{-\left(g_{t i j}\left(\left\langle\sigma_{\mathrm{ann}} v\right\rangle, J_{t}\right)+b_{t i j}\right)} \times \frac{\left(\tau_{t i} b_{t i j}\right)^{N_{\mathrm{OFF}, t i j}}}{N_{\mathrm{OFF}, t i j} !} e^{-\tau_{t i} b_{t i j}}\right] \\
& \times \mathcal{T}\left(\tau_{t i} \mid \tau_{\mathrm{obs}, t i}, \sigma_{\tau, t i}\right) \times \mathcal{J}\left(J_{t} \mid \log _{10} J_{\mathrm{obs}, t}, \sigma_{\log _{10} J, t}\right),
\end{aligned}
$$

where $v$ is the set of nuisance parameters, $J$ is the $J$-factor value, $b$ is the expected number of background events, $\tau$ is the $\mathrm{OFF} / \mathrm{ON}$ acceptance ratio, $N_{\mathrm{ON}}$ and $N_{\mathrm{OFF}}$ are the number of observed events in the ON region and the OFF region, respectively, and $g$ the expected number of signal events.

The likelihood function is written as the product of a Poisson function for $N_{\mathrm{ON}}$, one for $N_{\mathrm{OFF}}$, a Gaussian function for the $\tau$ parameter (with mean value $\tau_{\mathrm{obs}}$ and variance $\sigma_{\tau}^{2}$ ) and a Gaussian function for the $J$ parameter (with mean $\log _{10} J_{\mathrm{obs}}$ and variance $\sigma_{\log _{10} J}^{2}$ ). All these functions are expressed in terms of $j$, that runs over the number of energy bins $N_{\text {bins. }}$.

The flux of gamma rays, that is described as a function of the parameter of interest for the study 
$\left\langle\sigma_{\mathrm{ann}} v\right\rangle$, is included in the expression of $g$ as follows:

$$
g_{j}\left(\left\langle\sigma_{\mathrm{ann}} v\right\rangle, J\right)=T_{\mathrm{obs}} \int_{E_{\min , j}^{\prime}}^{E_{\mathrm{max}, j}^{\prime}} d E^{\prime} \int_{0}^{\infty} d E \frac{d \phi\left(\left\langle\sigma_{\mathrm{ann}} v\right\rangle, J\right)}{d E} A_{\mathrm{eff}}(E) G\left(E^{\prime} \mid E\right)
$$

where $T_{\mathrm{obs}}$ is the effective observation time (as reported in Table 1), $A_{\mathrm{eff}}$ the collection area and $G\left(E^{\prime} \mid E\right)$ the probability density function for an event to migrate from the true energy $E$ to the estimated energy $E^{\prime}$.

The likelihood analysis is implemented in the gLike tool [7] and cross-checked using the independent software package LklCom [8]. It has to be noticed that the entire analysis considers the spatial distribution of DM of each target and, thus, the respective gamma-ray emission distribution with respect to the center of the target. This has been done thanks to the use of the Donut Monte Carlo tool [9].

\section{Results and discussion}

The DM analyses performed on Draco and Coma Berenices dSphs did not show any evidence for gamma rays emitted from the targets. Consequently, we computed upper limits (UL) at the $95 \%$ CL on the velocity-averaged annihilation cross-section. The case of Segue 1 and Ursa Major II is similar, as presented in $[9,10]$.

A DM analysis was then performed on the combined data sample of $354.4 \mathrm{~h}$. UL at the $95 \% \mathrm{CL}$ have been set also in this case. Figure 1 shows the individual limits for the four $\mathrm{dSphs}$ and the limit obtained for the combined data set.

Even if the limits derived with the combined data sample are driven by the Segue 1 contribution, we stress that the weight of the systematic uncertainties related to the latter is reduced due to the
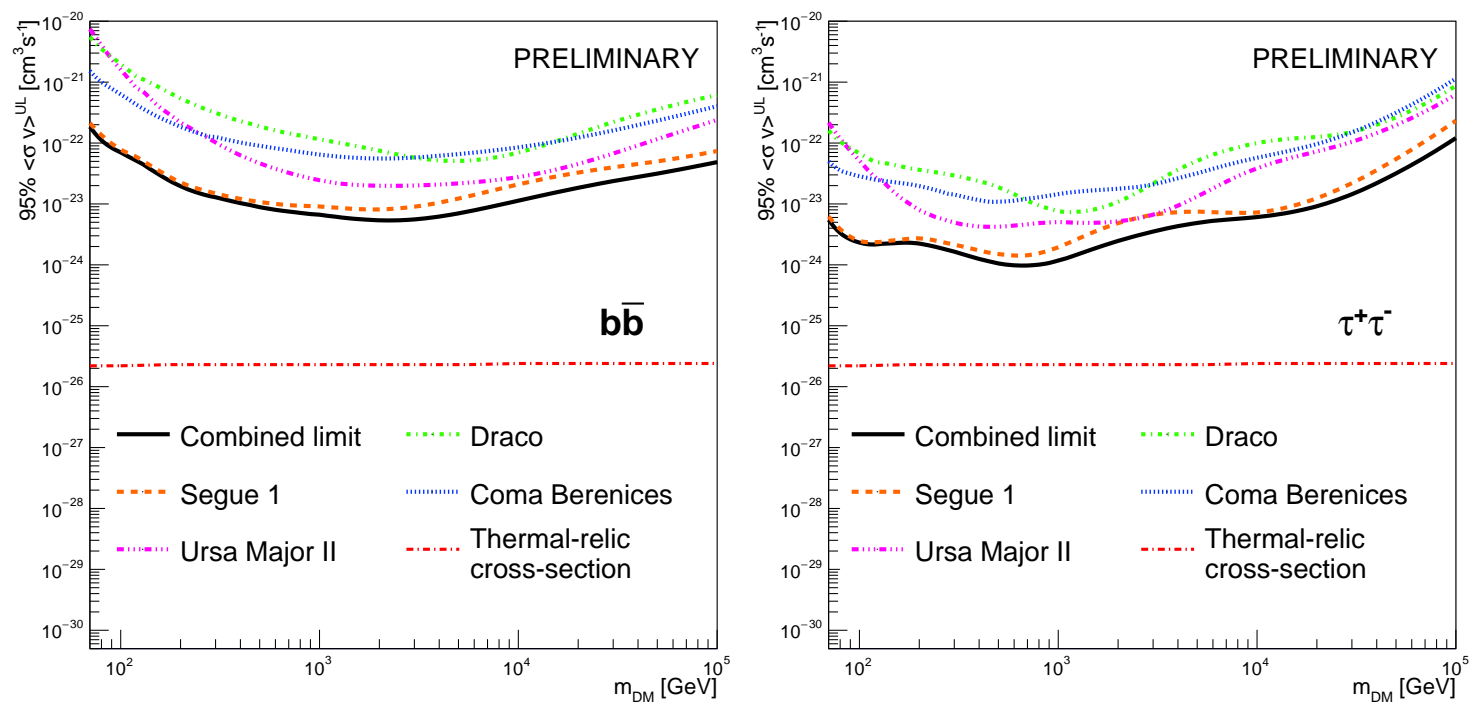

Figure 1: $95 \%$ CL ULs on the WIMP velocity-averaged annihilation cross-sections for the $b \bar{b}$ (left) and $\tau^{+} \tau^{-}$(right) channels, for the single dSphs (Segue 1-orange, Ursa Major II-violet, Draco dSph- light green, Coma Berenices dSph-blue) and for the data combination (solid black line). 
data combination.

The ULs presented are the most stringent ones within the MAGIC collaboration and very competitive with respect to other experiments, in particular in the TeV regime, such as Fermi-LAT, H.E.S.S., VERITAS and HAWC.

\section{Conclusions}

The MAGIC collaboration performed a multi-year observational diversification program to search for DM signals in dSphs, which are among the most promising targets for indirect DM searches. The program had the scope to diversify the dSph targets, in order to avoid possible biases in target selection, and accumulate dSphs data, allowing the improvement of previous results. For this reason, Draco dSph and Coma Berenices dSph were observed in 2018 and 2019, respectively. After the data analysis of both targets, no hint of DM signals were found and ULs at the 95\% CL on the velocity-averaged annihilation cross-section of WIMPs were set. In a second step, the data of these two dSphs were combined to the data of previously observed Segue 1 and Ursa Major II dSphs. This has been done in order to mitigate the effects from target related systematic uncertainties and improve the UL values obtained per each DM mass. The limits computed by the combination of $354.4 \mathrm{~h}$ of good quality data are about a factor 2 more stringent than the previously published MAGIC results and are the most constraining among current Cherenkov telescopes in the WIMP mass range from $70 \mathrm{GeV}$ to $100 \mathrm{TeV}$.

\section{Acknowledgements}

We acknowledge the support from the agencies and organizations listed here:

https://magic.mpp.mpg.de/acknowledgments_ICRC2021

\section{References}

[1] Springel, V. and Frenk, C. S. and White, S. D. M., The large-scale structure of the Universe, Nature 440 (2006) pp. 1137-1144 arXiv:astro-ph/0604561 [astro-ph]

[2] Aleksić, J. et al., The major upgrade of the MAGIC telescopes, Part II: A performance study using observations of the Crab Nebula, Astroparticle Physics 72 (2016) 76-94, arXiv:1409.5594 [astro-ph.IM]

[3] Feng, J. L., Dark Matter Candidates from Particle Physics and Methods of Detection, Annual Review of Astronomy and Astrophysics 48 (2010) 495-545, arXiv: 1003.0904

[4] Griest, K. and Kamionkowski, M., Unitarity limits on the mass and radius of dark-matter particles, Physical Review Letters, 64 (1990) 615-618

[5] Geringer-Sameth, A. and Koushiappas, S. M. and Walker, M., Dwarf Galaxy Annihilation and Decay Emission Profiles for Dark Matter Experiments, The Astrophysical Journal 801 (2015) 74, arXiv:1408.0002 [astro-ph.CO] 
[6] Zanin, R. et al., MARS, The MAGIC Analysis and Reconstruction Software, Proceedings, 33rd International Cosmic Ray Conference (ICRC2013) 33 (2013) 2937, https://ui.adsabs.harvard.edu/abs/2013ICRC...33.2937Z

[7] Rico, J. and Nigro, C. and Kerszberg, D. and Miener, T. and Aleksić, J., gLike: numerical maximization of heterogeneous joint likelihood functions of a common free parameter plus nuisance parameters, Zenodo (2021) v00.09.03, https://doi.org/10.5281/zenodo.4601451

[8] Miener, T. and Nieto D., LklCom: Combining likelihoods from different experiments., Zenodo (2021) v0.5.3, https://doi.org/10.5281/zenodo.4597500

[9] Ahnen, M. L. et al., Indirect dark matter searches in the dwarf satellite galaxy Ursa Major II with the MAGIC telescopes, Journal of Cosmology and Astroparticle Physics 3 (2018) 009, arXiv:1712.03095 [astro-ph.HE]

[10] Aleksić, J. et al., Optimized dark matter searches in deep observations of Segue 1 with MAGIC, Journal of Cosmology and Astroparticle Physics 2 (2014) 008, arXiv:1312.1535 [astro-ph.HE] 


\section{Full Authors List: MAGIC Collaboration}

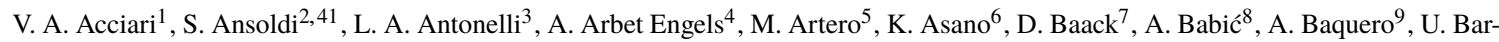
res de Almeida ${ }^{10}$, J. A. Barrio ${ }^{9}$, I. Batković ${ }^{11}$, J. Becerra González ${ }^{1}$, W. Bednarek ${ }^{12}$, L. Bellizzi ${ }^{13}$, E. Bernardini ${ }^{14}$, M. Bernardos ${ }^{11}$, A. Berti ${ }^{15}$, J. Besenrieder ${ }^{15}$, W. Bhattacharyya ${ }^{14}$, C. Bigongiari ${ }^{3}$, A. Biland ${ }^{4}$, O. Blanch ${ }^{5}$, H. Bökenkamp ${ }^{7}$, G. Bonnoli ${ }^{16}$, Ž. Bošnjak $^{8}$, G. Busetto ${ }^{11}$, R. Carosi ${ }^{17}$, G. Ceribella ${ }^{15}$, M. Cerruti ${ }^{18}$, Y. Chai ${ }^{15}$, A. Chilingarian ${ }^{19}$, S. Cikota ${ }^{8}$, S. M. Colak ${ }^{5}$, E. Colombo ${ }^{1}$, J. L. Contreras ${ }^{9}$, J. Cortina ${ }^{20}$, S. Covino ${ }^{3}$, G. D’Amico ${ }^{15,42}$, V. D’Elia ${ }^{3}$, P. Da Vela ${ }^{17,43}$, F. Dazzi ${ }^{3}$, A. De Angelis ${ }^{11}$, B. De Lotto ${ }^{2}$, M. Delfino ${ }^{5,44}$, J. Delgado ${ }^{5,44}$, C. Delgado Mendez ${ }^{20}$, D. Depaoli ${ }^{21}$, F. Di Pierro ${ }^{21}$, L. Di Venere ${ }^{22}$, E. Do Souto Espiñeira ${ }^{5}$, D. Dominis Prester $^{23}$, A. Donini ${ }^{2}$, D. Dorner ${ }^{24}$, M. Doro ${ }^{11}$, D. Elsaesser ${ }^{7}$, V. Fallah Ramazani ${ }^{25,45}$, A. Fattorini ${ }^{7}$, M. V. Fonseca ${ }^{9}$, L. Font ${ }^{26}$, C. Fruck ${ }^{15}$, S. Fukami ${ }^{6}$, Y. Fukazawa ${ }^{27}$, R. J. García López ${ }^{1}$, M. Garczarczyk ${ }^{14}$, S. Gasparyan ${ }^{28}$, M. Gaug ${ }^{26}$, N. Giglietto ${ }^{22}$, F. Giordano ${ }^{22}$, P. Gliwny ${ }^{12}$, N. Godinović ${ }^{29}$, J. G. Green ${ }^{3}$, D. Green ${ }^{15}$, D. Hadasch ${ }^{6}$, A. Hahn ${ }^{15}$, L. Heckmann ${ }^{15}$, J. Herrera ${ }^{1}$, J. Hoang ${ }^{9,46}$, D. Hrupec ${ }^{30}$, M. Hütten ${ }^{15}$, T. Inada ${ }^{6}$, K. Ishio ${ }^{12}$, Y. Iwamura ${ }^{6}$, I. Jiménez Martínez ${ }^{20}$, J. Jormanainen ${ }^{25}$, L. Jouvin ${ }^{5}$, M. Karjalainen ${ }^{1}$, D. Kerszberg ${ }^{5}$, Y. Kobayashi' ${ }^{6}$, H. Kubo ${ }^{31}$, J. Kushida ${ }^{32}$, A. Lamastra ${ }^{3}$, D. Lelas ${ }^{29}$, F. Leone ${ }^{3}$, E. Lindfors ${ }^{25}$, L. Linhoff ${ }^{7}$, S. Lombardi ${ }^{3}$, F. Longo ${ }^{2,47}$, R. López-Coto ${ }^{11}$, M. López-Moya ${ }^{9}$, A. López-Oramas ${ }^{1}$, S. Loporchio ${ }^{22}$, B. Machado de Oliveira Fraga $^{10}$, C. Maggio ${ }^{26}$, P. Majumdar ${ }^{33}$, M. Makariev ${ }^{34}$, M. Mallamaci ${ }^{11}$, G. Maneva ${ }^{34}$, M. Manganaro ${ }^{23}$, K. Mannheim ${ }^{24}$, L. Maraschi ${ }^{3}$, M. Mariotti ${ }^{11}$, M. Martínez ${ }^{5}$, D. Mazin ${ }^{6,48}$, S. Menchiari ${ }^{13}$, S. Mender ${ }^{7}$, S. Mićanović23, D. Miceli ${ }^{2,49}$, T. Miener ${ }^{9}$, J. M. Miranda ${ }^{13}$, R. Mirzoyan ${ }^{15}$, E. Molina ${ }^{18}$, A. Moralejo ${ }^{5}$, D. Morcuende ${ }^{9}$, V. Moreno ${ }^{26}$, E. Moretti ${ }^{5}$, T. Nakamori ${ }^{35}$, L. Nava ${ }^{3}$, V. Neustroev ${ }^{36}$, C. Nigro ${ }^{5}$, K. Nilsson ${ }^{25}$, K. Nishijima ${ }^{32}$, K. Noda $^{6}$, S. Nozaki ${ }^{31}$, Y. Ohtani ${ }^{6}$, T. Oka ${ }^{31}$, J. Otero-Santos ${ }^{1}$, S. Paiano ${ }^{3}$, M. Palatiello ${ }^{2}$, D. Paneque ${ }^{15}$, R. Paoletti ${ }^{13}$, J. M. Paredes ${ }^{18}$, L. Pavletić ${ }^{23}$, P. Peñil ${ }^{9}$, M. Persic ${ }^{2,50}$, M. Pihet ${ }^{15}$, P. G. Prada Moroni ${ }^{17}$, E. Prandini ${ }^{11}$, C. Priyadarshi ${ }^{5}$, I. Puljak ${ }^{29}$, W. Rhode ${ }^{7}$, M. Ribó ${ }^{18}$, J. Rico ${ }^{5}$, C. Righi ${ }^{3}$, A. Rugliancich ${ }^{17}$, N. Sahakyan ${ }^{28}$, T. Saito ${ }^{6}$, S. Sakurai ${ }^{6}$, K. Satalecka ${ }^{14}$, F. G. Saturni ${ }^{3}$, B. Schleicher ${ }^{24}$, K. Schmidt $^{7}$, T. Schweizer ${ }^{15}$, J. Sitarek ${ }^{12}$, I. Šnidarić ${ }^{37}$, D. Sobczynska ${ }^{12}$, A. Spolon ${ }^{11}$, A. Stamerra ${ }^{3}$, J. Strišković ${ }^{30}$, D. Strom ${ }^{15}$, M. Strzys ${ }^{6}$, Y. Suda ${ }^{27}$, T. Surić ${ }^{37}$, M. Takahashi ${ }^{6}$, R. Takeishi ${ }^{6}$, F. Tavecchio ${ }^{3}$,

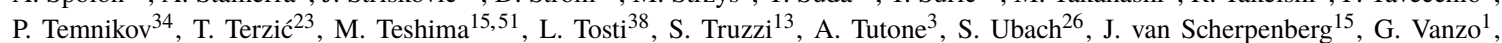
M. Vazquez Acosta ${ }^{1}$, S. Ventura ${ }^{13}$, V. Verguilov ${ }^{34}$, C. F. Vigorito ${ }^{21}$, V. Vitale ${ }^{39}$, I. Vovk $^{6}$, M. Will ${ }^{15}$, C. Wunderlich ${ }^{13}$, T. Yamamoto $^{40}$, and D. Zarić 29

${ }^{1}$ Instituto de Astrofísica de Canarias and Dpto. de Astrofísica, Universidad de La Laguna, E-38200, La Laguna, Tenerife, Spain. ${ }^{2}$ Università di Udine and INFN Trieste, I-33100 Udine, Italy. ${ }^{3}$ National Institute for Astrophysics (INAF), I-00136 Rome, Italy. ${ }^{4}$ ETH Zürich, CH-8093 Zürich, Switzerland. ${ }^{5}$ Institut de Física d'Altes Energies (IFAE), The Barcelona Institute of Science and Technology (BIST), E-08193 Bellaterra (Barcelona), Spain. ${ }^{6}$ Japanese MAGIC Group: Institute for Cosmic Ray Research (ICRR), The University of Tokyo, Kashiwa, 277-8582 Chiba, Japan. ${ }^{7}$ Technische Universität Dortmund, D-44221 Dortmund, Germany. ${ }^{8}$ Croatian MAGIC Group: University of Zagreb, Faculty of Electrical Engineering and Computing (FER), 10000 Zagreb, Croatia. ${ }^{9}$ IPARCOS Institute and EMFTEL Department, Universidad Complutense de Madrid, E-28040 Madrid, Spain. ${ }^{10}$ Centro Brasileiro de Pesquisas Físicas (CBPF), 22290-180 URCA, Rio de Janeiro (RJ), Brazil. ${ }^{11}$ Università di Padova and INFN, I-35131 Padova, Italy. ${ }^{12}$ University of Lodz, Faculty of Physics and Applied Informatics, Department of Astrophysics, 90-236 Lodz, Poland. ${ }^{13}$ Università di Siena and INFN Pisa, I-53100 Siena, Italy. ${ }^{14}$ Deutsches Elektronen-Synchrotron (DESY), D-15738 Zeuthen, Germany. ${ }^{15}$ Max-Planck-Institut für Physik, D-80805 München, Germany. ${ }^{16}$ Instituto de Astrofísica de Andalucía-CSIC, Glorieta de la Astronomía s/n, 18008, Granada, Spain. ${ }^{17}$ Università di Pisa and INFN Pisa, I-56126 Pisa, Italy. ${ }^{18}$ Universitat de Barcelona, ICCUB, IEEC-UB, E-08028 Barcelona, Spain. ${ }^{19}$ Armenian MAGIC Group: A. Alikhanyan National Science Laboratory, 0036 Yerevan, Armenia. ${ }^{20}$ Centro de Investigaciones Energéticas, Medioambientales y Tecnológicas, E-28040 Madrid, Spain. ${ }^{21}$ INFN MAGIC Group: INFN Sezione di Torino and Università degli Studi di Torino, I-10125 Torino, Italy. ${ }^{22}$ INFN MAGIC Group: INFN Sezione di Bari and Dipartimento Interateneo di Fisica dell'Università e del Politecnico di Bari, I-70125 Bari, Italy. ${ }^{23}$ Croatian MAGIC Group: University of Rijeka, Department of Physics, 51000 Rijeka, Croatia. ${ }^{24}$ Universität Würzburg, D-97074 Würzburg, Germany. ${ }^{25}$ Finnish MAGIC Group: Finnish Centre for Astronomy with ESO, University of Turku, FI-20014 Turku, Finland. ${ }^{26}$ Departament de Física, and CERES-IEEC, Universitat Autònoma de Barcelona, E-08193 Bellaterra, Spain. ${ }^{27}$ Japanese MAGIC Group: Physics Program, Graduate School of Advanced Science and Engineering, Hiroshima University, 739-8526 Hiroshima, Japan. ${ }^{28}$ Armenian MAGIC Group: ICRANet-Armenia at NAS RA, 0019 Yerevan, Armenia. ${ }^{29}$ Croatian MAGIC Group: University of Split, Faculty of Electrical Engineering, Mechanical Engineering and Naval Architecture (FESB), 21000 Split, Croatia. ${ }^{30}$ Croatian MAGIC Group: Josip Juraj Strossmayer University of Osijek, Department of Physics, 31000 Osijek, Croatia. ${ }^{31}$ Japanese MAGIC Group: Department of Physics, Kyoto University, 606-8502 Kyoto, Japan. ${ }^{32}$ Japanese MAGIC Group: Department of Physics, Tokai University, Hiratsuka, 259-1292 Kanagawa, Japan. ${ }^{33}$ Saha Institute of Nuclear Physics, HBNI, 1/AF Bidhannagar, Salt Lake, Sector-1, Kolkata 700064, India. ${ }^{34}$ Inst. for Nucl. Research and Nucl. Energy, Bulgarian Academy of Sciences, BG-1784 Sofia, Bulgaria. ${ }^{35}$ Japanese MAGIC Group: Department of Physics, Yamagata University, Yamagata 990-8560, Japan. ${ }^{36}$ Finnish MAGIC Group: Astronomy Research Unit, University of Oulu, FI-90014 Oulu, Finland. ${ }^{37}$ Croatian MAGIC Group: Ruđer Bošković Institute, 10000 Zagreb, Croatia. ${ }^{38}$ INFN MAGIC Group: INFN Sezione di Perugia, I-06123 Perugia, Italy. ${ }^{39}$ INFN MAGIC Group: INFN Roma Tor Vergata, I-00133 Roma, Italy. ${ }^{40}$ Japanese MAGIC Group: Department of Physics, Konan University, Kobe, Hyogo 658-8501, Japan. ${ }^{41}$ also at International Center for Relativistic Astrophysics (ICRA), Rome, Italy. ${ }^{42}$ now at Department for Physics and Technology, University of Bergen, NO-5020, Norway. ${ }^{43}$ now at University of Innsbruck. ${ }^{44}$ also at Port d'Informació Científica (PIC), E-08193 Bellaterra (Barcelona), Spain. ${ }^{45}$ now at Ruhr-Universität Bochum, Fakultät für Physik und Astronomie, Astronomisches Institut (AIRUB), 44801 Bochum, Germany. ${ }^{46}$ now at Department of Astronomy, University of California Berkeley, Berkeley CA 94720. ${ }^{47}$ also at Dipartimento di Fisica, Università di Trieste, I-34127 Trieste, Italy. ${ }^{48}$ Max-Planck-Institut für Physik, D-80805 München, Germany. ${ }^{49}$ now at Laboratoire d'Annecy de Physique des Particules (LAPP), 
CNRS-IN2P3, 74941 Annecy Cedex, France. ${ }^{50}$ also at INAF Trieste and Dept. of Physics and Astronomy, University of Bologna, Bologna, Italy. ${ }^{51}$ Japanese MAGIC Group: Institute for Cosmic Ray Research (ICRR), The University of Tokyo, Kashiwa, 277-8582 Chiba, Japan. 